\title{
D. Conhitz y L. Estrada-GonzÁlez. An Introduction to the Philosophy of Logic,
} Cambridge University Press, Cambridge /Nueva York, 2019, 265 páginas.

Este es un libro de indagación de límites. En la naturaleza de la lógica. Si se quiere comprender las posibles ampliaciones, si se trata de tener acceso a nuevos planteamientos, transformaciones esenciales y a la presencia de nuevos límites que parece experimentar hoy la lógica, ello se lo puede encontrar en dos ámbitos al menos: en los propios desarrollos de la lógica, especialmente desde mediados del siglo XX, pero, también, en la filosofía de la lógica. Cohnitz y Estrada-González ofrecen en este texto una muy actualizada aproximación introductoria del corrimiento de algunos de los límites de la disciplina que hoy se discuten en el segundo ámbito, en la filosofía de la lógica. Se trata de enfrentar al lector, hoy, a la naturaleza de lo lógico en cuanto tal, en aquellos temas y problemas que parecen estar transformando la disciplina desde aquella que se heredó de Frege.

Como los autores se encargan de aclarar en la Introducción, no se trata aquí ni de "metalógica", en cuanto disciplina que se ocupa de las propiedades estructurales de la relación de consecuencia válida, ni tampoco de "lógica filosófica", en tanto conjunto de sistemas formales que incorporan elementos del lenguaje y la argumentación que han preocupado a la filosofía, y que han determinado nuevas sistemas lógicos, como, por ejemplo, "existencia", "temporalidad", "vaguedad", "relevancia". No se trata, pues, de ninguno de los dos ámbitos mencionados simplemente porque ambos son, sin más, lógica propiamente tal y no filosofía de la lógica.

A diferencia de lo anterior, entonces, el libro, con un carácter también de apoyo al estudio (cada capítulo termina en un breve cuestionario sobre lo tratado), introduce en 10 capítulos los temas y problemas propiamente filosóficos que presenta la lógica contemporánea, específicamente las cuestiones de índole ontológica y epistemológica, de cuál es la naturaleza de lo lógico propiamente tal y de qué manera estamos ciertos de que una consecuencia lógica realmente lo es. En este sentido se trata de un texto que sigue la tradición filosófica acerca de la lógica que se encuentran en libros y artículos que ya se pueden considerar clásicos como son, por ejemplo, los de S. Read (1995), S. Haack (1996), R. I. Hughes (ed.), (1993) o D. Jacquette (2002). El libro trata de los problemas permanentes de la filosofía de la lógica: qué son y cómo se determinan las constantes lógicas; las relaciones complejas entre la lógica de primer orden y de orden superior; el análisis de los dos enfoques estándar actual de la lógica, la visión modelo-teórica y el enfoque de la prueba-teórica; la cuestión transversal de si la lógica es esencialmente normativa o acepta también elementos de descriptividad; el carácter preservador de la verdad en la deducción; la revisión del antipsicologismo, tal vez la marca de la lógica formal matemática a partir de Frege-Husserl; o qué es lo "formal" de la lógica formal, o, en fin, si la lógica es fundamentalmente a priori o no.

Si bien el lector encontrará en el libro un robusto tratamiento de estas y otras cuestiones ya clásicas de la filosofía de la lógica, muchas de ellas siempre aporéticas, el interés central del libro radica en lo que se podría denominar la ampliación de los límites de lo lógico que se viene observando en las últimas décadas. En tal sentido, este texto se emparenta con libros como los de Penélope Rush (edit. 2014) o de Stewart 
Shapiro (2014) o Beall y Restall (2006), por ejemplo, que presentan preocupaciones por ciertas cuestiones filosóficas que ensanchan o cambian la visión que se tiene de la lógica actualmente, o que al menos son proyectos de ello. Así, Cohnitz y EstradaGonzález ponen a la naturaleza de la lógica en una perspectiva de desarrollo, de temas aún en elaboración, que desafían las posturas más clásicas. Por supuesto que estos temas límites que se analizan, como se los podría denominar, no son todos los que hoy se pueden entender con ese rol y características, pero sí al menos son algunos de los que se pueden identificar como recurrentes e importantes de tratar. Por lo demás, esta cuestión estará siempre de suyo sujeta a polémica, pues debe reconocerse que no es de por sí claro qué temas puedan ser considerados ya clásicos o cuáles de nuevo cuño $\mathrm{y}$ con un rol de frontera.

Dentro del conjunto de aproximaciones de ampliación esencial de la lógica que ofrecen los autores, son destacables al menos cuatro de ellos: la cuestión de la lógica entendida como su aplicación canónica y su relación con el "mundo" (cap. 1); la cuestión de la revisabilidad de la lógica (cap. 6); el asunto del desarrollo actual del pluralismo lógico (cap.7) y la perspectiva cognitiva de la naturaleza de la lógica (caps. 5, 8 y10).

La distinción entre lógica "pura” y "aplicada” cobra importancia filosófica (así como hoy se diferencia entre ética aplicada y ética pura). Siguen los autores a G.Priest en la distinción mencionada. Pero también hay otra distinción en paralelo: entre la lógica como una teoría sobre ciertos objetos y lógica como lo estudiado por esa teoría. Como en la historia: como "hechos" y como historiografía. Pues bien, la distinción pura/aplicada es aplicable a "Cada uno de los miembros de la distinción teoría/objeto" (p. 13). Una lógica pura es una cierta estructura formal matemática, estudiada, por ejemplo, como "lógica universal". Por otro lado, la lógica aplicada, esto es no pura, es un determinado fenómeno que posee una estructura lógica subyacente. Un determinado fenómeno, no en sí lógico, puede ser comprendido a través de una estructura lógica. Así, por ejemplo, un circuito eléctrico, en sí ontológicamente muy alejado de la lógica, es analizado en su estructura subyacente como una lógica proposicional aplicada, en que constantes lógicas como la disyunción y la conjunción sirven para interpretar situaciones de "interruptores", en estados de abiertos o cerrados. O un sistema de control automático de sistemas fácticos que manejan gradualidades constituyen lógicas fuzzy aplicadas. Pero, dentro de las aplicaciones, hay una que es privilegiada, puesto que se la considera como del dominio propio de la lógica. A ella Priest la denominó aplicación canónica. La aplicación canónica de la lógica es la deducción.

Pues bien, lo anterior posee consecuencias e interrogantes para la concepción de la lógica. Primera: aunque los autores no lo explicitan, la deducción como tal, como operación que produce consecuencia lógica entre enunciados, no es parte de lo "puro" de la lógica; segunda, cabe la pregunta, ¿dónde radica esencialmente lo lógico, en lo puro o en lo aplicado? De ordinario se ha considerado a la deducción como lo esencial de la lógica y que la distingue de toda otra disciplina (incluso de la matemática). Pero esa tesis parece estar transformándose a la luz de esta taxonomía; tercero, ¿no se debería, entonces, considerar a los argumentos de la vida diaria (dominio de lo que se denomina "teoría de la argumentación") como parte de la lógica, en cuanto aplicada, en vez de centrarse en la disputa de si la lógica informal es o no lógica; cuarta implicación 
relevante de la taxonomía expuesta: lo que comúnmente entendemos por lógica (por ejemplo, la "conceptografía" de Frege) sería una aplicación canónica, no lógica pura. ¿Y qué son, entonces, los sistemas lógicos diversos actuales, aplicaciones canónicas? Lo que se denomina "lógica" sería propiamente tal la aplicación canónica de estructuras formales. La aplicación canónica lo que hace es interpretar la "teoría pura", la pura estructura formal en términos de "sentencias", "inferencias", "argumentos", "verdad", etc. Pues la lógica pura no contiene ningún concepto como los que tradicionalmente asociamos con lo lógico ¿Es, entonces, la lógica pura representante de lo lógico propiamente tal, como parecía inicialmente? Parece que la lógica es esencialmente la evaluación de la forma de los argumentos más que el estudio de ciertas estructuras. Afirman los autores al respecto:

Sin embargo, la mayoría de los lógicos, filósofos y aún matemáticos son todavía reticentes a la idea de considerar la lógica como un estudio formal de ciertas estructuras más que la ciencia de la evaluación de argumentos (p. 21).

Parece, pues, que lo natural sería considerar, todavía, la lógica como la aplicación canónica más que como una estructura formal donde incluso los conceptos mismos de inferencia y consecuencia están ausentes. En fin, la taxonomía explorada es de enorme utilidad para dar cuenta de otras cuestiones, como la del pluralismo lógico y la revisabilidad de la lógica.

Sobre el segundo punto de límites (uno de los problemas epistemológicos tratados en el cap. 6), se formula en la pregunta siguiente: ¿se puede considerar la lógica como una teoría, como una teoría fáctica que explica ciertos objetos? Esta idea parece alejarse bastante de la herencia lógica. Si una teoría empírica determinada lo es, lo es porque puede ser revisada, aún eliminada. Esa es su naturaleza. Ciencia empírica significa justamente eso. Pero la lógica, como disciplina formal, parecería lejos de ello, por definición independiente de cualquier determinación fáctica. ¿Es revisable, pues la lógica? Afirman los autores que este ha sido uno de los "tres dogmas" de la lógica: no es revisable, es a priori y no se la puede justificar. Los autores siguen aquí la propuesta desafiante de Priest: sí, la lógica es revisable, y debe ser revisada en una taxonomía básica que distingue tres formas: logica docens, logica utens y logica ens. La primera, la lógica "que se enseña", parece indudablemente revisable. Es un hecho que la lógica ha cambiado en su historia. Así, por ejemplo, sucede con el Darapti aristotélico: (i) Todo A es B; (ii) Todo A es C; (iii) Todo B es C, es válido dado que se asume la existencia de A. La lógica libre, hoy, en cambio considera que, para que sea válida esa inferencia, debe agregarse la cláusula de explicitación: ¡ $\boxminus A$, esto es, la cláusula: "siempre que exista A" (p. 149). Es evidente, pues, que tal estructura ha sido revisada hoy en día. Por su parte, la lógica utens presenta una situación menos clara. Se trata de la lógica con la que razonamos cotidianamente o en las ciencias. Se trata en realidad de algo así como de "prácticas inferenciales" y, en tal sentido, responden también a normas que pueden ser cambiadas (para Priest, debe aclararse, la logica utens no es descriptiva, como normalmente se la considera, sino que es normativa). Pero, afirman algunos críticos a esta idea, si un "método", como dice Field, resulta que es racionalmente revisable, quiere decir entonces que no resulta ser fundamental. Y 
la lógica es: "después de todo, nuestra metodología más fundamental” (p. 150). Mas, las críticas no alcanzan, según Cohnitz y Estrada-González, a cuestionar radicalmente la posibilidad de la revisabilidad de la lógica. Así, ejemplifican con la paradoja del mentiroso: "(a) la sentencia (a) es falsa". A la luz de la lógica estándar la paradoja es inaceptable. Si todas las soluciones a la paradoja fallan entonces consideraremos la revisión de la lógica estándar. Y tal revisión debe ser "recomendada" por esa lógica estándar, por lo que: "la lógica estándar falla en sus propios estándares" (p. 150).

El asunto de la lógica ens es mucho más complejo, puesto que se trata aquí de los mismos hechos que se siguen o no de qué otros hechos. Es una suerte de visión ontologizada de la lógica. Si se acepta, afirman los autores, una perspectiva de un realismo platónico de la lógica, esta trata con hechos lógicos independientes absolutamente de nosotros, de cómo razonamos y de lo que creamos acerca de cómo lo hacemos; "Si esto es así, entonces nosotros no podemos revisar esos hechos" (p. 148). Sin embargo, ejemplifican los autores, ello tampoco es demasiado claro y suficiente: el calentamiento global es un fenómeno independiente de nuestra mente, es un hecho del mundo, pero que, no obstante, no es, en principio al menos, algo que no se lo pueda "revisar", en el sentido de "cambiar", o de intentarlo al menos. Pero, la lógica sería, en esta perspectiva de lógica ens, tan fundamental y no fáctica que sería lo absolutamente aislado respecto de nosotros. Los autores hacen hincapié, en todo caso, que para Priest la lógica ens es también revisable (como esperaba Quine después de todo), como el calentamiento global, como todo por lo demás. Si los hechos lógicos, afirman siguiendo a Priest, son relaciones entre significados, como hechos abstractos, podemos cambiar y revisar qué significados expresan nuestras palabras.

La tercera cuestión es la del pluralismo lógico, tratado en el capítulo 7. La filosofía de la lógica se ha encaminado hacia una defensa del pluralismo frente a las posturas monistas. El problema no es de hecho, por supuesto; no se trata de reconocer que existen en la actualidad innumerables sistemas lógicos, clásicos, no clásicos, subestructurales, derrotables. El pluralismo es: "La visión de que existe más de una lógica correcta o, alternativamente, que hay más de una genuina relación de consecuencia” (p. 162).

Los autores recorren los diversos problemas y contraproblemas que se presentan en relación con esta cuestión: he allí a los defensores del monismo; el "principio de tolerancia" de Carnap en 1937, signado hoy como uno de los originadores del pluralismo; los diferentes tipos de pluralismo (punteado, local, distribuido y universal); las posturas pluralistas actuales, como la de Shapiro, un "pluralismo ecléctico" o la de Beall y Restall, un pluralismo "basado en caso".

¿Pero qué significa “correcto" en: "hay más de una lógica correcta”? Según los autores, la postura de Beall y Restall remite a Tarski: la lógica debe tener las características relevantes que el concepto intuitivo de consecuencia, de razonamiento correcto, en el lenguaje natural posee. El problema en síntesis es: ¿Cómo una teoría $\mathrm{T}_{1}$ puede afirmar que $\mathrm{B} \vDash \mathrm{A} v \neg \mathrm{A}$ y otra teoría $\mathrm{T}_{2}$, afirmar que $\mathrm{B} \vDash \mathrm{Av} \neg \mathrm{A}, \mathrm{y}$, sin embargo, $\mathrm{T}_{1} \mathrm{y}_{2}$ ser ambas correctas? Para Carnap la cuestión era que la diferencia de consecuencia lógica remitía a una cuestión de diferencia de lenguaje adoptado, libremente adoptado. Pero hoy esto ya no es así. La respuesta de Shapiro, por ejemplo, es que, si se estudia 
una estructura que posee una lógica subyacente que no es clásica (por ejemplo, en los fenómenos físicos cuánticos), es más conveniente estudiarla en su propia lógica subyacente no clásica (que no posee ciertas propiedades clásicas) que el esfuerzo de tratar de traducir esa lógica a términos clásicos. El resultado sería manifiestamente forzado. La postura de Shapiro posee el interés especial de que se concibe a la lógica como una teoría que "modela" los argumentos naturales y, en tal sentido, esencialmente no tiene por qué existir solo un modelo correcto (es esencial a la idea misma de "modelo" en sentido cognitivo el ser una representación de ciertos elementos estructurales que son función de lo que se requiere representar, por lo que no existe un modelo perfecto ni un único modelo adecuado para lo modelado). Esto levanta un asunto relacionado con la cuestión de la distinción "pura/aplicada" de la lógica. ¿No es en la lógica aplicada donde se daría el pluralismo?

La postura de Beall y Restall, por su parte, es esencialmente la siguiente: hay más de una relación genuina de consecuencia, que no provienen de diferentes lenguajes, sino que emergen de las exigencias de un mismo lenguaje. Como afirman Cohnitz y Estrada-González: "Logic(s) is (are) concerned with a single area, namely the evaluation of arguments; it is the same (form of) argument in the same language which is evaluated in differents cases" (p. 168). En síntesis, esta visión es un pluralismo de los distintos sentidos que puede adoptar la idea de consecuencia lógica. La base estaría en la tesis general de Tarski de consecuencia lógica, según la cual un argumento (determinado) es válido si y solo si cada caso de argumento, si tiene premisas verdaderas, no tiene conclusión falsa (p. 168). Se trata de una suerte de "plantilla" para determinar la validez de casos de argumentos (la relación verdad-no falsedad, por lo demás, proviene de lejos en la historia de la lógica). Pero esto indica un significado especial de "seguirse de". Y no es el único significado, esa es la cuestión del pluralismo según Beall y Restall. Al sentido tarskianio de validez recién mencionado hay otro, dentro del mismo lenguaje, hay que recalcarlo (con lo que se supera la visión de Carnap), y es el de considerar que la validez tiene que ver con la exigencia de que las premisas y la conclusión tengan alguna relación entre sí. De este modo, si en el primer sentido de consecuencia, $A^{\wedge} \neg A$ $\vDash B$ es válido, en el segundo sentido manifiestamente no lo es. Entonces: si no existe una sola concepción de consecuencia, el pluralismo lógico es sostenible.

En los capítulos 5, 8 y 10, los autores exponen uno de los temas tal vez más relevantes para la comprensión de la "ampliación" actual del sentido de la lógica: la relación entre lógica y ciencia cognitiva. El peligro que se ha visto en esta relación es su reminiscencia al "psicologismo"; pero la relación con la cognición no es psicologismo y ello es lo que los autores esclarecen. La relación entre lógica y cognición es, pues, el ámbito donde cobra mayor notoriedad el corrimiento de límites de la naturaleza de la lógica, pues esta entra en un campo que la herencia fregeana había prohibido. (Cabe observar, en todo caso, que, sobre este tema, la postura asumida por la mayoría de los autores es la pregunta por cómo la lógica puede determinar la cognición; mas, hay también otra relación, la inversa, que normalmente no se asume: la que interroga por cuánto la ciencia cognitiva puede determinar la naturaleza de la lógica. Es el problema central de una "filosofía cognitiva de la lógica". R. Hanna, cap. 5, es una excepción). 
Dado que lógica y ciencia cognitiva poseen una evidente intersección, el razonamiento como fenómeno humano, la relación entre ambas disciplinas no es una ilusión ni algo descaminado. Un primer asunto es, entonces, qué relación guardan lógica y razonamiento. El libro analiza en detalle las diferentes posturas sobre la siguiente interrogante: ¿Puede ser la lógica normativa respecto del razonamiento cotidiano, entendido este como hecho cognitivo, tales como lo son la percepción, la memoria, etc.? Entiéndese, pues, que el problema proviene de que el "razonar" propiamente tal no es un concepto lógico sino cognitivo. La tesis clásica, por decirlo así, es negativa y está representada, con distinta fuerza, por ejemplo por las tesis de G. Harman, L. Cosmides o P. Johnson-Laird. El razonamiento común no puede ser normado por las leyes de la lógica (véase las cuatro críticas de Harman expuestas por los autores, en p. 183 y ss.). Cohnitz y Estrada-González defienden la postura positiva; la lógica sí puede cumplir roles normativos respecto del razonamiento. Las posturas en defensa de la tesis afirmativa son expuestas sobre la base de las posiciones de F. Steinberger, quien desafía a Harman, específicamente. En el cap. 10 los autores siguen la siguiente argumentación: la postura como la de Harman se sustenta en general en la conocida experiencia llevada a cabo por Wason en 1966, en la llamada tarea de selección, que muestra que las personas no razonan en dicha tarea con el modus tollens. De allí en más se asumió que la lógica no puede tener ninguna relación de normatividad sobre el razonamiento en situaciones corrientes. Cohnitz y Estrada-González defienden, en todo caso, que no todo razonamiento común está de hecho determinado normativamente por la lógica, sino que la postura es más débil: se constata que hay roles normativos. Los autores afirman que la crítica a la tarea de selección que han hecho Stennings y van Lambalgen es clara en este sentido. La mayoría de la psicología del razonamiento ha supuesto que la regla lógica que se evalúa en la tarea de Wason "posee una forma lógica no ambigua" (p. 228). Pero eso es cuestionable. Pues no parece ser estrictamente la misma regla la que actúa en las dos partes del experimento, en su versión abstracta (solo con letras y números) y la versión de control con contenido fáctico (con situaciones de la vida diaria). En el caso abstracto el condicional que enuncia la tarea "Es mejor interpretado como descriptivo" (p. 228); en tanto, en el caso no abstracto: "parece tratarse de un condicional deóntico" (p. 228). Y esto hace que, en el segundo caso, al ser una "orden" el condicional que instruye la tarea, es más fácilmente entendible para el sujeto del experimento.

Cohnitz y Estrada-González, pues, al inicio del cap. 8, argumentan que, si el razonamiento pretende llegar a creencias verdaderas sobre el "mundo", creencias que poseen un "contenido proposicional" con relaciones lógicas entre sí, y si el proyecto de la ciencia cognitiva es la verdad de las creencias, tendremos que mostrar interés también en las relaciones lógicas que tengan providencia en esas relaciones entre creencias, que son cogniciones (p. 182). Es relevante notar aquí la alusión al "mundo", que no es por cierto el de la facticidad diaria o científica sino que es un "mundo argumentativo", por el que interpretamos el mundo fáctico. 
Referencias bibliográficas

Beall J y G. Restall (2006), Logical Pluralism. Oxford: Clarendon Press.

Haack S. (1996), Fuzzy Logic, Deviant Logic. Beyond the Formalism. Chicago, Londres: The University of Chicago Press.

Hughes R. I. (1993), edit., A Philosophical Companion to First-Order-Logic Indianapolis Cambridge: Hackett Publishing Company.

Jacquette D, (2002), edit., Philosophy of Logic. Malden, Oxford: Blackwell Publishers. Read S. (1995), Thinking about Logic. Oxford, N.York, Oxford University Press.

Rush P. (2014), edit., The metaphysics of Logic. Cambridge: Cambridge University Press.

Shapiro S. (2014), Varieties of Logic. Oxford: Oxford University Press.

Alejandro RAmÍREZ Universidad de Chile alramire@uchile.cl 